\title{
Program Verification using Constraint Handling Rules and Array Constraint Generalizations $\rrbracket^{*}$
}

\author{
Emanuele De Angelis ${ }^{1}$, Fabio Fioravanti ${ }^{1}$, \\ Alberto Pettorossi ${ }^{2}$, and Maurizio Proietti ${ }^{3}$ \\ 1 DEC, University 'G. D'Annunzio', Pescara, Italy, \{emanuele.deangelis, fioravanti\}@unich.it \\ ${ }^{2}$ DICII, University of Rome Tor Vergata, Rome, Italy, pettorossi@disp.uniroma2.it \\ 3 IASI-CNR, Rome, Italy, maurizio.proietti@iasi.cnr.it
}

\begin{abstract}
The transformation of constraint logic programs (CLP programs) has been shown to be an effective methodology for verifying properties of imperative programs. By following this methodology, we encode the negation of a partial correctness property of an imperative program prog as a predicate incorrect defined by a CLP program $P$, and we show that prog is correct by transforming $P$ into the empty program through the application of semantics preserving transformation rules. Some of these rules perform replacements of constraints that encode properties of the data structures manipulated by the program prog. In this paper we show that Constraint Handling Rules (CHR) are a suitable formalism for representing and applying constraint replacements during the transformation of CLP programs. In particular, we consider programs that manipulate integer arrays and we present a CHR encoding of a constraint replacement strategy based on the theory of arrays. We also propose a novel generalization strategy for constraints on integer arrays that combines the CHR constraint replacement strategy with various generalization operators for linear constraints, such as widening and convex hull. Generalization is controlled by additional constraints that relate the variable identifiers in the imperative program prog and the CLP representation of their values. The method presented in this paper has been implemented and we have demonstrated its effectiveness on a set of benchmark programs taken from the literature.
\end{abstract}

\section{Introduction}

It has long been recognized that Constraint Logic Programming (CLP) is a formalism that provides very suitable inference mechanisms for the verification of properties of imperative programs. The landmark paper [41] has shown that: (i) the operational semantics of imperative programs can easily be formalized as an interpreter written in CLP, and (ii) by specializing that interpreter with respect to a given imperative program, say prog, one can derive a new CLP program, say $V C$, representing the verification conditions for prog in purely logical form. In particular, in the specialized CLP program $V C$ there are no references to the imperative constructs of prog. Relevant properties of the execution of prog (such as its loop invariants) can then be inferred by analyzing the program $V C$.

Many verification methods within the CLP paradigm have been developed. Some methods, directly following the approach presented in [41, are based on abstract interpretation [8] and compute an overapproximation of the least model of the CLP program under consideration by a bottom-up evaluation of an abstraction of the program [2, 28, 39]. Other methods use goal directed evaluation of CLP programs combined with other symbolic techniques such as interpolation [17, 20, 31, 30. Some other methods, like the ones presented in [5, 25, 43, 45],

*This work has been partially supported by the National Group of Computing Science (GNCS-INDAM). 
combine CLP (also called constrained Horn clauses in those papers) with different reasoning techniques developed in the areas of Software Model Checking and Automated Theorem Proving, such as CounterExample-Guided Abstraction Refinement (CEGAR) and Satisfiability Modulo Theory (SMT).

In this paper we follow the approach based on transformations of CLP programs presented in [12, 14]. We encode the negation of a partial correctness property of an imperative program prog as a predicate incorrect defined by a CLP program $P$. Similarly to [41, we generate a CLP program $V C$ representing the verification conditions for prog, by specializing $P$ with respect to the CLP representation of prog. However, at this point the transformation-based method departs from the ones considered above. Indeed, it continues by applying further equivalence preserving transformations to $V C$ with the objective of deriving either (i) the empty CLP program, hence proving that incorrect does not hold and prog is correct, or (ii) a CLP program containing the fact incorrect, hence proving that prog is incorrect. Due to the undecidability of partial correctness, it may be the case that we derive a CLP program containing one or more clauses of the form incorrect :- G, where $\mathrm{G}$ is a non-empty conjunction, and we are able to conclude neither that prog is correct nor that prog is incorrect.

Thus, CLP program transformation provides a uniform framework for reasoning about the correctness of imperative programs in which, as we have explained, one can generate the verification conditions and also check their validity. Moreover, that framework is parametric with respect to the syntax and the semantics of the programs to be verified, and optimizing transformations considered in the literature [42] can be applied to improve the efficiency of the verification method. Finally, transformations can easily be composed together into a sequence of transformations, so as to derive very sophisticated verification methods. For instance, in [13] it is shown that the iteration of program specialization can significantly improve the precision of our program verification method and indeed, by implementing Iterated Specialization the VeriMAP system [15] is competitive with state-of-the-art CLP-based verifiers such as ARMC [43], HSF [25], and TRACER [30].

The main contributions of this paper are the following.

(1) We consider imperative programs that manipulate integers and integer arrays, and we generate verification conditions where read and write operations on arrays are represented as constraints. Then we show that Constraint Handling Rules (CHR) are a suitable formalism for manipulating constraints during the transformation of the CLP verification conditions. In particular, we present CHR rules based on the theory of arrays [7, 23, 37] and we show how they can be combined with unfold/fold transformation rules for CLP programs [18] with the objective of proving properties of the given imperative programs.

(2) We propose a powerful transformation strategy that guides the application of both the CHR and the unfold/fold transformation rules. In particular, we design a novel array constraint generalization strategy that automatically introduces, during CLP transformation, the new predicate definitions (corresponding to program invariants) required for the verification of the properties of interest. Our generalization strategy combines CHR manipulation of array constraints with the widening and convex hull operators for linear constraints considered in the field of abstract interpretation [10. Generalization is controlled by means of additional constraints that relate the variable identifiers in the given imperative programs and the CLP representations of their values.

(3) Finally, we present an implementation of the method in the VeriMAP system [15], and we demonstrate its effectiveness on a set of benchmark programs taken from the literature. 


\section{The Transformation-Based Verification Method}

In this section we introduce a class of Constraint Logic Programs with constraints on integers and integer arrays, and we show how partial correctness properties of imperative programs can be encoded as programs of that class.

First we need the following definitions. An atomic integer constraint is either $\mathrm{p}_{1}=\mathrm{p}_{2}$, or $\mathrm{p}_{1} \geq \mathrm{p}_{2}$, or $\mathrm{p}_{1}>\mathrm{p}_{2}$, where $\mathrm{p}_{1}$ and $\mathrm{p}_{2}$ are linear polynomials with integer variables and coefficients (sum and multiplication are denoted by + and $*$, respectively). An atomic array constraint is either $\operatorname{dim}(\mathrm{a}, \mathrm{n})$ denoting that the dimension of the array a is $\mathrm{n}$, or $\operatorname{read}(\mathrm{a}, \mathrm{i}, \mathrm{v})$ denoting that the $i$-th element of the array a is the integer $v$, or write $(a, i, v, b)$ denoting that the array $b$ is equal to the array a, except that its i-th element is $v$. The read and write constraints satisfy the following axioms [7, 23], where variables are universally quantified at the front:
(A1) $\mathrm{I}=\mathrm{J}, \operatorname{read}(\mathrm{A}, \mathrm{I}, \mathrm{U}), \quad \operatorname{read}(\mathrm{A}, \mathrm{J}, \mathrm{V}) \rightarrow \mathrm{U}=\mathrm{V}$
(array congruence)
$(\mathrm{A} 2) \mathrm{I}=\mathrm{J}, \operatorname{write}(\mathrm{A}, \mathrm{I}, \mathrm{U}, \mathrm{B}), \operatorname{read}(\mathrm{B}, \mathrm{J}, \mathrm{V}) \rightarrow \mathrm{U}=\mathrm{V}$
(read-over-write: case $=$ )
$(\mathrm{A} 3) \mathrm{I} \neq \mathrm{J}, \operatorname{write}(\mathrm{A}, \mathrm{I}, \mathrm{U}, \mathrm{B}), \operatorname{read}(\mathrm{B}, \mathrm{J}, \mathrm{V}) \rightarrow \operatorname{read}(\mathrm{A}, \mathrm{J}, \mathrm{V})$ (read-over-write: case $\neq$ )

A constraint is either true, or an atomic (integer or array) constraint, or a conjunction of constraints. An atom is a formula of the form $\mathrm{p}\left(\mathrm{t}_{1}, \ldots, \mathrm{t}_{\mathrm{m}}\right)$, where $\mathrm{p}$ is a predicate symbol not in $\{=, \geq,>$, dim, read, write $\}$ and $t_{1}, \ldots, t_{m}$ are terms constructed out of variables, constants, and function symbols different from + and $*$. A CLP program is a finite set of clauses of the form $\mathrm{A}:-\mathrm{C}, \mathrm{B}$, where $\mathrm{A}$ is an atom, $\mathrm{C}$ is a constraint, and $\mathrm{B}$ is a (possibly empty) conjunction of atoms. Given a clause A :- c, B, the atom A is called the head, and c, B is called the body. We assume that in every clause all integer arguments in its head are distinct variables. A clause A : - $\mathrm{c}$ is called a constrained fact. If $\mathrm{c}$ is true, then it is omitted and the constrained fact is called a fact. A CLP program is said to be linear if all its clauses are of the form A :- c, B, where $\mathrm{B}$ consists of at most one atom.

An $\mathcal{A}$-interpretation $I$ is a set $D$, together with a function $f$ in $D^{n} \rightarrow D$ for each function symbol $\mathrm{f}$ of arity $n$, and a relation $p$ on $D^{n}$ for each predicate symbol $\mathrm{p}$ of arity $n$, such that: (i) the set $D$ is the Herbrand universe 36 constructed out of the set $\mathbb{Z}$ of the integers, the constants, and the function symbols different from + and (ii) $I$ assigns to the symbols $+, *,=, \geq$, > the usual meaning in $\mathbb{Z}$, (iii) for all sequences $a_{0} \ldots a_{n-1}$, for all integers $\mathrm{d}, \operatorname{dim}\left(\mathrm{a}_{0} \ldots \mathrm{a}_{\mathrm{n}-1}, \mathrm{~d}\right)$ is true in $I$ iff $\mathrm{d}=\mathrm{n}$, (iv) for all sequences $\mathrm{a}_{0} \ldots \mathrm{a}_{\mathrm{n}-1}$ and $\mathrm{b}_{0} \ldots \mathrm{b}_{\mathrm{m}-1}$ of integers, for all integers $i$ and $v, \operatorname{read}\left(a_{0} \ldots a_{n-1}, i, v\right)$ is true in $I$ iff $0 \leq i \leq n-1$ and $\mathrm{v}=\mathrm{a}_{\mathrm{i}}$, and write $\left(\mathrm{a}_{0} \ldots \mathrm{a}_{\mathrm{n}-1}, \mathrm{i}, \mathrm{v}, \mathrm{b}_{0} \ldots \mathrm{b}_{\mathrm{m}-1}\right)$ is true in $I$ iff $0 \leq \mathrm{i} \leq \mathrm{n}-1, \mathrm{n}=\mathrm{m}, \mathrm{b}_{\mathrm{i}}=\mathrm{v}$, and for $j=0, \ldots, n-1$, if $j \neq i$ then $a_{j}=b_{j}$, (v) $I$ is an Herbrand interpretation [36] for function and predicate symbols different from $+, *,=, \geq,>$, dim, read, and write.

We can identify an $\mathcal{A}$-interpretation $I$ with the set of all ground atoms that are true in $I$, and hence $\mathcal{A}$-interpretations are partially ordered by set inclusion. A constraint $\mathrm{c}$ is said to be satisfiable if $\mathcal{A}=\exists(\mathrm{c})$, where in general, for every formula $\varphi, \exists(\varphi)$ denotes the existential closure of $\varphi$. We say that $I$ is an $\mathcal{A}$-model of $\varphi$ if $\varphi$ is true in $I$. We write $\mathcal{A} \models \varphi$ if every $\mathcal{A}$-interpretation is an $\mathcal{A}$-model of $\varphi$. In particular, every $\mathcal{A}$-interpretation is an $\mathcal{A}$-model of Axioms (A1)-(A3). A constraint c entails a constraint d, denoted $\mathrm{c} \sqsubseteq \mathrm{d}$, if $\mathcal{A} \models \forall(\mathrm{c} \rightarrow \mathrm{d})$. By $\operatorname{vars}(\varphi)$ we denote the free variables of $\varphi$. The semantics of a CLP program $P$ is the least $\mathcal{A}$-model of $P$, denoted $M(P)$ and constructed as usual for CLP programs [29].

We consider imperative programs with integer and array variables. Every program has a single halt command whose execution causes the program to terminate. The semantics of programs is defined in terms of a transition relation, denoted $\Longrightarrow$, between configurations. A configuration is a pair $\langle\langle c, \delta\rangle$ of a labeled command $c$ and an environment $\delta$ that maps: (i) every integer variable identifier $x$ to its value $v$, and (ii) every integer array identifier $a$ to a finite 
sequence $\mathrm{a}_{0} \ldots \mathrm{a}_{n-1}$ of integers, where $\mathrm{n}$ is the dimension of the array $a$. The transition relation specifies the 'small step' operational semantics and its definition is similar to that in [44] and is omitted. An environment $\delta$ is said to satisfy a formula $\varphi\left(z_{1}, \ldots, z_{r}\right)$ iff $\varphi\left(\delta\left(z_{1}\right), \ldots, \delta\left(z_{r}\right)\right)$ holds.

Given two formulas $\varphi_{\text {init }}$ and $\varphi_{\text {error }}$ that are disjunctions of constraints with free variables $z_{1}, \ldots, z_{r}$, we say that program prog is incorrect with respect to these formulas iff there exist two environments $\delta_{\text {init }}$ and $\delta_{h}$ such that: (i) $\delta_{\text {init }}$ satisfies $\varphi_{\text {init }}$, (ii) $\left\langle\ell_{0}: c_{0}, \delta_{\text {init }}\right\rangle \Longrightarrow^{*}\left\langle\left\langle\ell_{h}\right.\right.$ : halt, $\left.\delta_{h}\right\rangle$, and (iii) $\delta_{h}$ satisfies $\varphi_{\text {error }}$, where $\ell_{0}: c_{0}$ is the first labeled command of prog and $\ell_{h}$ : halt is the unique halt command of prog. A program is said to be correct if it is not incorrect. (In [1] the reader may find an extension of these definitions in the case where $\varphi_{\text {init }}$ and $\varphi_{\text {error }}$ are predicates defined by any CLP program.) Our notion of correctness is equivalent to the Hoare notion of partial correctness specified by the triple $\left\{\varphi_{\text {init }}\right\} \operatorname{prog}\left\{\neg \varphi_{\text {error }}\right\}$.

We translate the problem of checking whether or not the program prog is incorrect into the problem of checking whether or not the atom incorrect is a consequence of the following CLP program $T$ :

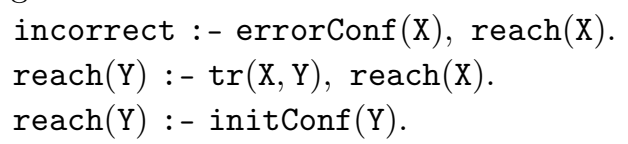

where initConf $(\mathrm{X})$, errorConf $(\mathrm{X})$, and $\operatorname{tr}(\mathrm{X}, \mathrm{Y})$ are defined by CLP clauses so that the following conditions hold. For all configurations $\mathrm{X}$ and $\mathrm{Y}$, (i) initConf $(\mathrm{X})$ holds iff $\mathrm{X}$ is an initial configuration, that is, a configuration of the form $\left\langle\ell_{0}: c_{0}, \delta_{\text {init }}\right\rangle$ and $\delta_{\text {init }}$ satisfies $\varphi_{\text {init }}$, (ii) errorConf $(\mathrm{X})$ holds iff $\mathrm{X}$ is an error configuration, that is, a configuration of the form $\left\langle\left\langle\ell_{h}\right.\right.$ : halt,$\left.\delta_{h}\right\rangle$ and $\delta_{h}$ satisfies $\varphi_{\text {error }}$, and (iii) $\operatorname{tr}(\mathrm{X}, \mathrm{Y})$ holds iff $\mathrm{X} \Longrightarrow \mathrm{Y}$ holds.

reach $(\mathrm{Y})$ holds iff the configuration $\mathrm{Y}$ can be reached from a configuration $\mathrm{X}$ whose environment satisfies $\varphi_{\text {init }}$. Program prog is correct with respect to $\varphi_{\text {init }}$ and $\varphi_{\text {error }}$ iff incorrect $\notin M(T)$.

Our verification method applies unfold/fold rules to the initial program $T$ and consists of following two steps 14. (i) VCGen: the Generation of the Verification Conditions, and (ii) VCTransf: the Satisfiability Checking of the Verification Conditions. The soundness of our method follows from the fact that for each program $U$ obtained from $T$ by applying the unfold/fold rules, incorrect $\in M(T)$ iff incorrect $\in M(U)$.

$V C G e n$ performs a specialization of program $T$ with respect to the given predicates tr (which depends on prog), initConf, and errorConf, thereby deriving a new program $T 1$, whose clauses are said to be the verification conditions for prog, such that $\operatorname{tr}$ does not occur in $T 1$ (for this reason this step is also called the removal of the interpreter). During this specialization step all occurrences of the dim predicate are replaced by suitable constraints on the indexes of the arrays. We say that the verification conditions are satisfiable iff incorrect $\notin M(T 1)$, and thus their satisfiability guarantees that prog is correct with respect to $\varphi_{\text {init }}$ and $\varphi_{\text {error }}$. The satisfiability of the verification conditions generated at the end of VCGen is checked by $V C T r a n s f$, which will be described in detail in Section 3.

Before starting the specialization, VCGen adds to the initial program $T$ some additional constraints that are needed for controlling the generalization strategy described in Section 3.3 These constraints use the predicate val that relates some of the variable identifiers occurring in the imperative program prog to the CLP representation of their values. In particular, the meaning of the val constraints is as follows: for every variable identifier $i$ of the program prog, for every value $\mathrm{I}$, the constraint val $(\mathrm{i}, \mathrm{I}$ ) (where $\mathrm{i}$ is a constant uniquely associated with $i$ ) holds iff there exists a configuration whose environment $\delta$ maps $i$ to I. These val constraints will be used by our generalization strategy to distinguish among different read constraints, thereby making the strategy more effective, as confirmed by the experimental results reported in Section 4 . For 
instance, the constraint ' $\operatorname{val}(i, I), \operatorname{val}(j, J), \operatorname{read}(A, I, U), \operatorname{read}(A, J, V)$ ' expresses the property that the first read gets the array element at index $i$ and the second read gets the array element at index $j$, while this property is not expressed by the constraint ' $\operatorname{read}(\mathrm{A}, \mathrm{I}, \mathrm{U}), \operatorname{read}(\mathrm{A}, \mathrm{J}, \mathrm{V})$ ' (without the val constraints), as the scope of the variable identifiers I and $\mathrm{J}$ is the clause where they occur.

Now, let us see our verification method in action on a simple example. Let us consider the following program that, given the array $a[0 . .(n-1)]$ and any $i \in\{0, \ldots, n-1\}$ places in $a[n-i-1]$ the maximum value of the leftmost portion $a[0 . .(n-i-1)]$ by iteratively swapping adjacent elements.

$$
\begin{aligned}
\text { bubblesort-inner: } & \text { for }(j=0 ; \quad j<n-i-1 ; \quad j++)\{ \\
& \quad \text { if }(a[j]>a[j+1])\{\operatorname{tmp}=a[j] ; \quad a[j]=a[j+1] ; \quad a[j+1]=t m p ;\}\}
\end{aligned}
$$

Let us also consider the two properties $\varphi_{\text {init }}(i, n, a) \equiv 0 \leq i<n \wedge \operatorname{dim}(a, n)$ and $\varphi_{\text {error }}(i, j, n, a) \equiv \exists k \exists x \exists y 0 \leq i<n \wedge 0 \leq k<j \wedge j=n-i-1 \wedge \operatorname{read}(a, k, x) \wedge \operatorname{read}(a, j, y) \wedge x>y$.

The two properties $\varphi_{\text {init }}$ and $\varphi_{\text {error }}$ are expressed in CLP as follows:

phinit $(\mathrm{I}, \mathrm{N}, \mathrm{A}):-0 \leq \mathrm{I}, \mathrm{I}<\mathrm{N}, \operatorname{dim}(\mathrm{A}, \mathrm{N})$.

phiError(I, J, N, A ) : - 0 I I, I $<\mathrm{N}, 0 \leq \mathrm{K}, \mathrm{K}<\mathrm{J}, \mathrm{J}=\mathrm{N}-\mathrm{I}-1, \mathrm{X}>\mathrm{Y}, \operatorname{read}(\mathrm{A}, \mathrm{K}, \mathrm{X}), \operatorname{read}(\mathrm{A}, \mathrm{J}, \mathrm{Y})$, $\operatorname{val}(k, K), \operatorname{val}(j, J)$.

Note the two val constraints that relate the index variables $k$ and $j$ to their values $\mathrm{K}$ and $\mathrm{J}$, respectively. At the end of VCGen we get the following CLP program T1 that expresses the verification conditions for the program bubblesort-inner:

1. incorrect : $-0 \leq \mathrm{I}, 0 \leq \mathrm{K}, \mathrm{K} \leq \mathrm{J}, \mathrm{J}=\mathrm{N}-\mathrm{I}-1, \mathrm{X}>\mathrm{Y}$, $\operatorname{read}(A, K, X), \operatorname{read}(A, J, Y), \overline{\operatorname{val}(k, K), \operatorname{val}(j, J), \operatorname{new1}(I, J, N, A, T m p, K) . ~}$

2. new1 $(\mathrm{I}, \mathrm{J} 1, \mathrm{~N}, \mathrm{~A} 2, \mathrm{~W}, \mathrm{~K}):-\mathrm{J} 1=1+\mathrm{J}, \mathrm{J}<\mathrm{N}-\mathrm{I}-1, \mathrm{~J} \geq 0, \mathrm{~J}<\mathrm{N}-1, \mathrm{X}>\mathrm{Y}$, $\operatorname{read}(A, J, X), \operatorname{read}(A, J 1, Y), \operatorname{read}(A, J, W), \operatorname{read}(A, J 1, Z), \overline{w r i t e}(A, J, Z, A 1)$, $\operatorname{write}(A 1, J 1, W, A 2), \operatorname{val}(j, J 1), \operatorname{val}(j, J), \operatorname{val}(k, K)$, new1(I, J, N, A,Tmp, K).

3. new1 $(\mathrm{I}, \mathrm{J} 1, \mathrm{~N}, \mathrm{~A}, \mathrm{Tmp}, \mathrm{K}):-\mathrm{J} 1=\mathrm{J}+1, \mathrm{~J}<\mathrm{N}-\mathrm{I}-1, \mathrm{~J} \geq 0, \mathrm{~J}<\mathrm{N}-1, \mathrm{X} \leq \mathrm{Y}$, $\operatorname{read}(A, J, X), \operatorname{read}(A, J 1, Y), \operatorname{val}(j, J 1), \operatorname{val}(j, J), \operatorname{val}(k, K), \overline{\operatorname{new} 1}(I, J, N, A, T m p, K)$.

4. new1 $(I, J, N, A, T m p, K):-0 \leq I, I<N, \underline{J}=0, \operatorname{val}(j, J), \operatorname{val}(k, K)$.

where new1 is a new predicate symbol introduced during program specialization by VCGen. The definition of the predicate new1 is associated with the for-loop of the bubblesort-inner program and consists of clauses 2-4 that represent the execution of the for statement. We have that (see the underlined constraints): (i) clauses 1 and 4 represent the exit and the entry of the for-loop, respectively, and (ii) clauses 2 and 3 represent the execution of the conditional in the $a[j]>a[j+1]$ case and in the $a[j] \leq a[j+1]$ case, respectively.

\section{A Transformation Strategy for Verification}

The VCTransf step of our verification method transforms the CLP program T1 derived at the end of VCGen to a program $T 2$ such that incorrect $\in M(T 1)$ iff incorrect $\in M(T 2)$. This transformation makes use of transformation rules that preserve the least $\mathcal{A}$-model semantics of CLP programs. In particular, we apply the following rules, that are collectively called unfold/fold rules: unfolding, constraint replacement, clause removal, definition, and folding. These rules are an adaptation to CLP programs of the unfold/fold rules for general CLP programs (see, for instance, [18]).

$V C T r a n s f$ applies the unfold/fold rules according to a strategy that performs the propagation of the constraints of the error property phiError in a backward way from the error 
configuration towards the initial configuration, so as to derive a program $T 2$ where the predicate incorrect is defined by either (i) the fact incorrect (in which case the imperative program prog is incorrect), or (ii) the empty set of clauses (in which case prog is correct). In the case where neither (i) nor (ii) holds, that is, in program $T 2$ the predicate incorrect is defined by a non-empty set of clauses not containing the fact incorrect, we cannot conclude anything about the correctness of prog. However, similarly to what has been proposed in [12, in this case we can perform again VCTransf by propagating the constraints of the initial property phiInit, and continue alternating the propagation of the constraints of the error property and the initial property, in the hope of deriving a program where either (i) or (ii) holds. Obviously, due to the undecidability of program correctness, it may be the case that this process does not terminate.

\subsection{The Transformation Strategy}

VCTransf is performed by applying the unfold/fold transformation rules according to the Transform strategy shown in Figure1. Let us briefly describe the various rules used by the Transform strategy.

- The Unfolding rule performs one step of backward propagation of the error property phiError.

- The Constraint Replacement rule infers new constraints on the variables of the single atom that occurs in the body of each clause obtained by Unfolding. Constraint RePLACEMENT makes use of a function Repl that, given a clause $C$ of the form $\mathrm{H}:-\mathrm{c}_{0}, \mathrm{~B}$, returns a set $\left\{\mathrm{H}:-\mathrm{C}_{1}, \mathrm{~B}, \ldots, \mathrm{H}:-\mathrm{C}_{\mathrm{n}}, \mathrm{B}\right\}$ of clauses (with $\mathrm{n} \geq 0$ ), where $\mathrm{c}_{1}, \ldots, \mathrm{c}_{\mathrm{n}}$ are constraints such that $\mathcal{A} \models \forall\left(\left(\exists \mathrm{X}_{0} \mathrm{c}_{0}\right) \leftrightarrow\left(\exists \mathrm{X}_{1} \mathrm{c}_{1} \vee \ldots \vee \exists \mathrm{X}_{\mathrm{n}} \mathrm{c}_{\mathrm{n}}\right)\right)$ holds, and for $i=0, \ldots, \mathrm{n}$, we have that $\mathrm{X}_{\mathrm{i}}=\operatorname{vars}\left(\mathrm{c}_{\mathrm{i}}\right)-\operatorname{vars}(\mathrm{H}, \mathrm{B})$. In particular, if $\mathrm{c}_{0}$ is unsatisfiable, then $\mathrm{n}=0$ and clause $C$ is removed. The function Repl is implemented by a CHR program as described in Section 3.2 .

- The rules of Removal of Useless Clauses and Removal of Subsumed Clauses remove clauses that do not contribute to the least model of the CLP program at hand.

- The Definition rule introduces new predicate definitions by suitable generalizations of the constraints. Generalization is performed by using a function $G e n$ such that, for any given clause $E$ of the form H:-e(V,X), $\mathrm{p}(\mathrm{X})$ and set Defs of predicate definitions, Gen(E,Defs) is a clause of the form newq $(X)$ :- $\operatorname{gen}(X), p(X)$, where: (i) newq is a new predicate symbol, and (ii) $\operatorname{gen}(\mathrm{X})$ is a constraint such that $\mathrm{e}(\mathrm{V}, \mathrm{X}) \sqsubseteq \operatorname{gen}(\mathrm{X})$.

- The Folding rule replaces the clause $H:-e(V, X), p(X)$ by the clause $H:-e(V, X)$, newq $(X)$.

Note that the input program $T 1$ of the Transform strategy is a linear CLP program. Indeed, during $V C G e n$ the atoms different from reach are unfolded and hence a linear program is generated.

The new predicates introduced by the DEFINITION rule can be understood as over-approximations of the sets of configurations that are backward-reachable from the error configuration. Note, however, that the folding rule preserves equivalence, as $\mathrm{e}(\mathrm{V}, \mathrm{X}), \mathrm{p}(\mathrm{X})$ is equivalent to $\mathrm{e}(\mathrm{V}, \mathrm{X})$, newq $(\mathrm{X})$. In Section 3.3 we will present a generalization function that guarantees the termination of Transform and, at the same time, allows us to prove the correctness of non-trivial programs.

We assume that the set Defs is structured as a tree of clauses where, with reference to Figure 1. clause $C$ is said to be the parent of clause $\operatorname{Gen}(E, D e f s)$, and the ancestor relation is defined as the reflexive, transitive closure of the parent relation. 
Input: A linear CLP program T1.

Output: Program $T 2$ such that incorrect $\in M(T 1)$ iff incorrect $\in M(T 2)$.

INITIALIZATION:

Let InDefs be the set of all clauses of $T 1$ whose head is the atom incorrect;

$T 2:=\emptyset ; \quad$ Defs $:=$ InDefs;

while in InDefs there is a clause $C$ of the form $\mathrm{H}:-\mathrm{c}, \mathrm{A} d o$

UNFOLDING: Let $\left\{\mathrm{K}_{i}:-\mathrm{c}_{i}, \mathrm{~B}_{i} \mid i=1, \ldots, m\right\}$ be the set of the (renamed apart) clauses of $T 1$ such that, for $i=1, \ldots, m, \mathrm{~A}$ is unifiable with $\mathrm{K}_{i}$ via the most general unifier $\vartheta_{i}$. Then TransfC $:=\left\{\left(\mathrm{H}:-\mathrm{c}, \mathrm{c}_{i}, \mathrm{~B}_{i}\right) \vartheta_{i} \mid i=1, \ldots, m\right\}$;

Constraint Replacement: TransfC $:=\cup_{D \in \operatorname{TransfC}} \operatorname{Repl}(D)$;

Removal of Subsumed Clauses: Remove from TransfC every clause $\mathrm{H}:-\mathrm{d}, \mathrm{B}$ such that there exists a distinct clause $\mathrm{H}:-\mathrm{e}$ in TransfC with $\mathrm{d} \sqsubseteq \mathrm{e}$;

DeFinition \& Folding:

while in TransfC there is a clause $E$ of the form $\mathrm{H}:-\mathrm{e}(\mathrm{V}, \mathrm{X}), \mathrm{p}(\mathrm{X})$, where $\mathrm{e}(\mathrm{V}, \mathrm{X})$ is a constraint and $\mathrm{p}$ is a predicate defined in $T 1 d o$

if in Defs there is a clause $D$ of the form newp (X) :- c (X), $\mathrm{p}(\mathrm{X})$, where $\mathrm{c}(\mathrm{X})$ is a constraint such that $\mathrm{e}(\mathrm{V}, \mathrm{X}) \sqsubseteq \mathrm{c}(\mathrm{X})$

then TransfC $:=($ TransfC $-\{E\}) \cup\{\mathrm{H}:-\mathrm{e}(\mathrm{V}, \mathrm{X})$, newp $(\mathrm{X})\}$;

else let $\operatorname{Gen}(E, D e f s)$ be newq(X):-gen(X), p(X);

Defs $:=\operatorname{Defs} \cup\{\operatorname{Gen}(E, D e f s)\}$;

InDefs $:=($ InDefs $-\{C\}) \cup\{\operatorname{Gen}(E$, Defs $)\}$;

TransfC $:=($ TransfC $-\{E\}) \cup\{\mathrm{H}:-\mathrm{e}(\mathrm{V}, \mathrm{X})$, newq $(\mathrm{X})\}$

end-while;

$T 2:=T 2 \cup \operatorname{TransfC}$

end-while;

Removal of Useless Clauses:

Remove from $T 2$ all clauses with head predicate $\mathrm{p}$, if in $T 2$ there is no constrained fact $q(\ldots)$ : - c where $q$ is either $p$ or a predicate on which $p$ depends.

Figure 1: The Transform strategy.

\subsection{Constraint Replacement via CHR}

In this section we show how Constraint Handling Rules with disjunction can be used to realize in a very natural way the constraint rewritings based on Axioms (A1)-(A3) for array operations, which allow us to apply the CONSTRAINT REPLACEMENT rule during the Transform strategy.

CHR is a committed-choice language based on rewriting rules. It was specifically designed for building custom constraint solvers 22. A CHR program consists of a set of guarded rules that rewrite multisets of constraints. Constraint predicates are of two kinds: (i) built-in constraints, whose entailment is checked by using a domain-specific constraint solver, and (ii) user-defined constraints, which are rewritten as specified by the CHR program. We assume that the set of built-in constraints contains the constraints true, false, and syntactic equalities. Built-in constraints and user-defined constraints are closed under conjunction. A constraint goal is either a (built-in or user-defined) constraint, or a conjunction of constraint goals, or a disjunction of constraint goals. 
CHR rules are of the form: $\mathrm{r} @ \mathrm{H}_{1} \backslash \mathrm{H}_{2}<=\mathrm{G} \mid \mathrm{B}$, where the @ symbol separates the optional rule identifier $\mathrm{r}$ from the rest of the rule, the user-defined constraints $\mathrm{H}_{1}$ and $\mathrm{H}_{2}$ are the kept head and the removed head, respectively, the built-in constraint $\mathrm{G}$ is the guard, and B is a constraint goal. Either $\mathrm{H}_{1}$ or $\mathrm{H}_{2}$ is a non-empty conjunction. If $\mathrm{H}_{2}$ is empty then the rule is called a propagation rule and can be written as follows: $\mathrm{H}_{1} \Rightarrow \mathrm{G} \mid \mathrm{B}$. The logical meaning of the CHR rule $\mathrm{H}_{1} \backslash \mathrm{H}_{2} \Leftrightarrow \mathrm{G} \mid \mathrm{B}$ is the guarded equivalence $\forall\left(\mathrm{G} \rightarrow\left(\left(\mathrm{H}_{1} \wedge \mathrm{H}_{2}\right) \leftrightarrow\left(\mathrm{H}_{1} \wedge \exists \mathrm{Y} \mathrm{B}\right)\right)\right)$, where $\mathrm{Y}$ is the set of all variables occurring in $\mathrm{B}$ and not in the rest of the rule.

The operational semantics of CHR is formally defined in terms of a transition relation between CHR states as described in [1]. A CHR state is a triple $\langle\mathrm{g}, \mathrm{u}, \mathrm{b}\rangle$, where $\mathrm{g}$ is a constraint goal, $\mathrm{u}$ is a user-defined constraint and $\mathrm{b}$ is a built-in constraint. An initial state is a state of the form $\langle\mathrm{g}$, true, true $\rangle$. Starting from an initial state, constraints are rewritten as long as possible by applying CHR rules. A final state is a state from which no transition is applicable. A final state is failed if it is of the form $\langle\mathrm{g}, \mathrm{u}, \mathrm{false}\rangle$. Note that, since constraint goals may contain disjunctions, the transition relation is nondeterministic, and thus it generates a tree of computations whose leaves correspond to the final states. A terminating CHR program is one for which there is no infinite sequence of transitions, that is, the tree of computations is finite.

The CHR program Arr used for constraint replacement in the Transform strategy consists of the following rules:

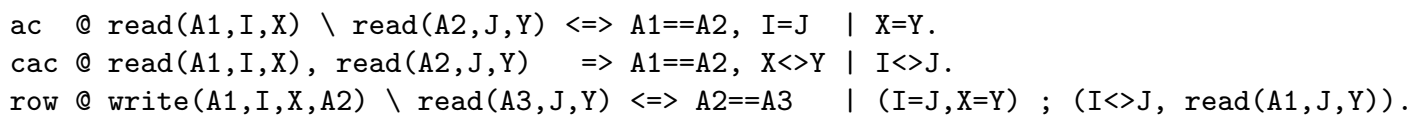

These rules encode the axioms (A1)-(A3) presented in Section 2 Rules ac and cac encode the array congruence axiom (A1) and its contrapositive version, respectively, and rule row encodes the two so-called read-over-write axioms (A2) and (A3). The symbol '==' denotes syntactic equality, while '=' and '<>' denote integer equality and inequality, respectively. Note that we use the semicolon ';' for denoting disjunction in the right-hand side of the rule row.

If we adopt an operational semantics that prevents trivial non-termination cases by applying a propagation rule at most once to the same constraints [1, then it can be shown that the CHR program Arr terminates for all constraint goals generated during the application of our transformation strategy. The proof is as follows. The only rule that may lead to a nonterminating behavior is row. By using this rule, a constraint containing

(g1) $\operatorname{write}(U, I, X, V), \operatorname{write}(V, I, H, U), \operatorname{read}(V, J, Y)$

could be rewritten as a constraint containing

(g2) $\operatorname{write}(U, I, X, V)$, $\operatorname{write}(V, I, H, U), \operatorname{read}(U, J, Y)$

and then, by interchanging the roles of the two write constraints in the application of the row rule, a constraint containing (g2) could be rewritten to a constraint containing (g1), thereby giving rise to an infinite branch in the tree of computation. However, it can be shown that a constraint goal of the form (g1) cannot be generated by the UNFOLDING rule during the application of the Transform strategy. Informally, in every clause, the constraints can be ordered from left to right, following the order of execution of the corresponding read and write operations, and hence a variable $\mathrm{V}$ occurring in a constraint of the form write(U, I, X, V), does not occur to the left of that constraint. This argument is formalized by considering the transitive closure $\prec^{+}$of the following relation between the variables of a clause: $\mathrm{U} \prec \mathrm{V}$ iff the constraint write(U, I, X, V) occurs in the clause. It can be shown that in every clause derived by the UNFOLDING rule during the application of the Transform strategy, $\prec^{+}$is irreflexive. Thus, the termination of Arr follows from the fact that an application of the row rule will replace a constraint of the form $\operatorname{read}(\mathrm{V}, \mathrm{J}, \mathrm{Y})$ by a constraint of the form $\operatorname{read}(\mathrm{U}, \mathrm{J}, \mathrm{Y})$ with $\mathrm{U} \prec \mathrm{V}$. 
Given a clause $D$ of the form $\mathrm{H}:-\mathrm{d}, \mathrm{B}$, derived by the UNFOLDING rule, let $\left\{\left\langle\mathrm{g}_{1}, \mathrm{u}_{1}, \mathrm{~b}_{1}\right\rangle, \ldots\right.$, $\left.\left\langle\mathrm{g}_{\mathrm{n}}, \mathrm{u}_{\mathrm{n}}, \mathrm{b}_{\mathrm{n}}\right\rangle\right\}$ be the set of all non-failed final states computed from the initial state $\langle\mathrm{d}$, true, true $\rangle$. Let $d_{i}$ be the conjunction $\left\langle g_{i}, u_{i}, b_{i}\right\rangle$. We assume that, for $i=1, \ldots, n$, the variables occurring in $d_{i}$ and not in $d$ are fresh, and thus they occur neither in $H$ nor in B. By the soundness of CHR we have that $\mathcal{A} \models \forall\left(\mathrm{d} \leftrightarrow\left(\exists \mathrm{x}_{1} \mathrm{~d}_{1} \vee \ldots \vee \exists \mathrm{X}_{\mathrm{n}} \mathrm{d}_{\mathrm{n}}\right)\right)$ where, for $\mathrm{i}=1, \ldots, \mathrm{n}, \mathrm{X}_{\mathrm{i}}=\operatorname{vars}\left(\mathrm{d}_{\mathrm{i}}\right)-\operatorname{vars}(\mathrm{d})$. Thus, the applicability conditions of the Constraint REPLACEMENT rule are satisfied, and in the Transform strategy we define $\operatorname{Repl}(D)$ to be $\left\{\mathrm{H}:-\mathrm{d}_{1}, \mathrm{~B}, \ldots, \mathrm{H}:-\mathrm{d}_{\mathrm{n}}, \mathrm{B}\right\}$.

To see how the CHR program Arr works, let us consider again the bubblesort-inner example of Section 3 . By applying the UNFOLDING rule to clause 1 the Transform strategy derives a set of clauses including the following one:

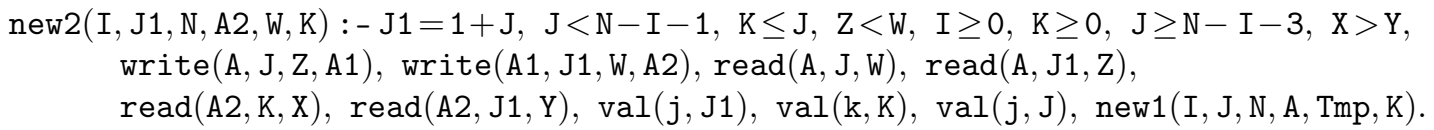

The CHR program Arr rewrites the constraint occurring in the above clauses and the CONSTRAINT REPLACEMENT rule derives the following clause:

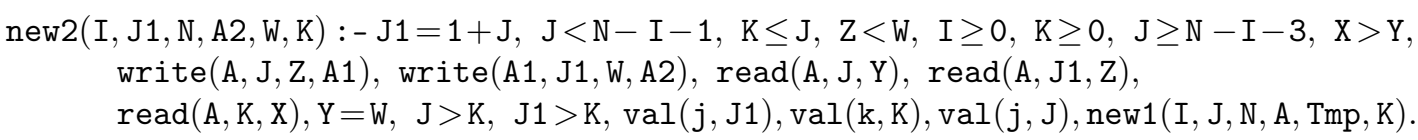

where (i) by row, the constraint $\operatorname{read}(\mathrm{A} 2, \mathrm{~J} 1, \mathrm{Y})$ has been replaced by the equality constraint $\mathrm{Y}=\mathrm{W}$ (ii) by row, in the constraint $\operatorname{read}(\mathrm{A} 2, \mathrm{~K}, \mathrm{X})$, the variable $\mathrm{A} 2$, denoting the array $a$ after the write operation, has been replaced by the variable $A$, denoting the array $a$ before the write operation, and (iii) the constraint ' $\mathrm{J}>\mathrm{K}, \mathrm{J} 1>\mathrm{K}$ ' has been added by the built-in solver on linear constraints.

\subsection{Generalization Strategy}

The most critical step of the Transform strategy is the introduction of new predicates during DEFINITION \& Folding. Indeed, it should guaranteed that a finite number of new predicates is introduced, to avoid the non-termination of Transform. For this reason, as usual in many program transformation techniques [19, we collect in the set Defs all predicate definitions introduced by the strategy, and before introducing a new predicate definition $D$, we match $D$ against the ones already in Defs. If $D$ is 'similar' to a definition $A$ in Defs (similarity is formalized via the embedding relation defined below), then the function Gen introduces a new definition which is a generalization of $A$ and $D$, instead of $D$. The function Gen defined in this section, makes use of operators for generalizing array constraints that ensure that no infinite number of distinct generalizations can be performed, and hence a finite number of new predicates is introduced during the Transform strategy. The embedding relation and the generalization strategy take into consideration the val constraints that relate the integer CLP variables occurring in read constraints and the identifiers of the imperative program with which they are associated. By doing so we will be able to identify similarities between definitions that go beyond syntactic variance, hence improving the level of precision of the verification technique.

In the following we will denote constraints as conjunctions of the form $i, r, w, v$, where $i$ is an integer constraint, and $r$, $w$, and $\mathrm{v}$ are conjunctions of read, write, and val constraints, respectively. We assume that all integer variables in read constraints are distinct and do not occur in any (non constraint) atom of the clause at hand (this condition can always be satisfied by adding some integer equalities).

Given a clause $D$ of the form $\mathrm{H}:-\mathrm{i}, \mathrm{r}, \mathrm{w}, \mathrm{v}, \mathrm{B}$, for every integer variable I occurring in a read atom in $\mathrm{r}$ we compute the set $i d s(\mathrm{I})$ of identifiers id such that an atom val(id, J) occurs 
in $\mathrm{v}$ and the constraint $\mathrm{I}=\mathrm{J}$ is entailed by $\mathrm{i}$. We define the clause identifier set of $D$, denoted $i d s(D)$, as the set of pairs $(i d s(\mathrm{I}), i d s(\mathrm{U}))$ such that a constraint of the form read(A, I, U) occurs in $r$. For example, if the constraint occurring in the body of clause $D$ is

$\mathrm{M}=0, \mathrm{~N}>\mathrm{M}, \mathrm{V}=0, \operatorname{read}(\mathrm{A}, \mathrm{M}, \mathrm{U}), \operatorname{read}(\mathrm{A}, \mathrm{N}, \mathrm{V}), \operatorname{val}(\mathrm{m}, \mathrm{M}), \operatorname{val}(\mathrm{n}, \mathrm{N}), \operatorname{val}(\mathrm{v}, \mathrm{V})$

then we have that $i d s(D)=\{(\{\mathrm{m}, \mathrm{v}\},\{\}),(\{\mathrm{n}\},\{\mathrm{m}, \mathrm{v}\})\}$.

Given two clause identifier sets $R_{1}$ and $R_{2}$, we say that $R_{1}$ is embedded into $R_{2}$ via the set relation rel iff for each pair $\left(I_{1}, U_{1}\right)$ in $R_{1}$ there exists a pair $\left(I_{2}, U_{2}\right)$ in $R_{2}$ such that: (i) $\operatorname{rel}\left(I_{1}, I_{2}\right)$ and $\operatorname{rel}\left(U_{1}, U_{2}\right)$ hold, and (ii) $R_{1}-\left\{\left(I_{1}, U_{1}\right)\right\}$ is embedded into $R_{2}-\left\{\left(I_{2}, U_{2}\right)\right\}$ via $\mathrm{rel}$. In our experiments we have considered two embedding relations based on the following definitions of $\operatorname{rel}\left(s_{1}, s_{2}\right):(1) s_{1} \subseteq s_{2}$ (subset relation), and (2) $s_{1} \cap s_{2}$ defined as $\left(s_{1}=s_{2}=\right.$ $\emptyset) \vee\left(s_{1} \cap s_{2} \neq \emptyset\right)$. We say that a clause $D_{1}$ is embedded into a clause $D_{2}$ via the relation rel iff $i d s\left(D_{1}\right)$ is embedded in $i d s\left(D_{2}\right)$ via rel.

Given a clause $E$ of the form $\mathrm{H}:-\mathrm{e}(\mathrm{V}, \mathrm{X}), \mathrm{p}(\mathrm{X})$ and a set Defs of definitions, the generalization function Gen computes a definition newq $(X):-\operatorname{gen}(X), p(X)$, where newq is a new predicate symbol and gen $(\mathrm{X})$ is a constraint such that $\mathrm{e}(\mathrm{V}, \mathrm{X}) \sqsubseteq \operatorname{gen}(\mathrm{X})$, which is constructed as follows. Let $\mathrm{e}(\mathrm{V}, \mathrm{X})$ be of the form $\mathrm{i}, \mathrm{r}, \mathrm{w}, \mathrm{v}$ and let newq $(\mathrm{X}):-\mathrm{i}_{\mathrm{X}}, \mathrm{r}_{\mathrm{X}}, \mathrm{v}_{\mathrm{X}}, \mathrm{p}(\mathrm{X})$ be the candidate definition clause for $E$, where: (i) $r_{\mathrm{X}}$ is the conjunction of the $\operatorname{read}(\mathrm{A}, \mathrm{I}, \mathrm{V})$ constraints in $\mathrm{r}$ such that $\mathrm{A}$ occurs in $\mathrm{X}$ and, for some $\operatorname{val}(\mathrm{j}, \mathrm{J})$ in $\mathrm{V}$ we have that $\mathrm{J}$ occurs in $\mathrm{X}$ and either $\mathrm{I}=\mathrm{J}$ or $\mathrm{V}=\mathrm{J}$ is entailed by $\mathrm{i}$, (ii) $i_{\mathrm{X}}$ is the constraint obtained from $\mathrm{i}$ by projecting away the variables not occurring in $\mathrm{X}$ or $r_{\mathrm{X}}$, and (iii) $\mathrm{v}_{\mathrm{X}}$ is the conjunction of the $\mathrm{val}(\mathrm{j}, \mathrm{J})$ constraints in $\mathrm{v}$ such that $\mathrm{J}$ occurs in $\mathrm{X}$.

Suppose that clause $E$ has been derived from clause $C$ at the end of the Removal of Subsumed Clauses step. Gen $(E, D e f s)$ is defined as follows.

If in Defs there is an ancestor $A$ of $C$ of the form $\mathrm{H}_{0}$ :- $\mathrm{i}_{0}, \mathrm{r}_{0}, \mathrm{v}_{0}, \mathrm{p}(\mathrm{X})$, such that $\mathrm{r}_{0}$ is a subconjunction of $\mathrm{r}_{\mathrm{X}}$, and $A$ is embedded into newq(X) :- $\mathrm{i}_{\mathrm{X}}, \mathrm{r}_{\mathrm{X}}, \mathrm{v}_{\mathrm{X}}, \mathrm{p}(\mathrm{X})$,

Then let $i_{1}$ be the constraint obtained from $i_{\mathrm{X}}$ by projecting away the variables not occurring in $\mathrm{X}$ or $\mathrm{r}_{0}$; compute a generalization $\mathrm{g}$ of the constraints $i_{1}$ and $i_{0}$ such that $i_{1} \sqsubseteq \mathrm{g}$, by using a generalization operator for linear constraints. Define the constraint gen $(\mathrm{X})$ as $\mathrm{g}, \mathrm{r}_{0}, \mathrm{v}_{0}$;

Else define the constraint $\operatorname{gen}(\mathrm{x})$ as $\mathrm{i}_{\mathrm{X}}, \mathrm{r}_{\mathrm{X}}, \mathrm{v}_{\mathrm{X}}$.

For the projection and generalization operations we apply the usual operators for linear constraints on the reals (and in particular the widening and convex hull generalization operators defined in [10, 19, 40]). These operators are correct because they guarantee that $i \sqsubseteq g$.

To see an example of application of the generalization strategy let us consider the clause that was derived in Section 3.2 by applying the Constraint Replacement rule. The candidate definition for that clause is:

new4 $(I, J, N, A, T m p, K):-J<N-I-1, I \geq 0, K \geq 0, J \geq N-I-3, X>W, J>K$, $\operatorname{read}(A, J, W), \operatorname{read}(A, K, X), \operatorname{val}(k, K), \operatorname{val}(j, J), \operatorname{new1}(I, J, N, A, T m p, K)$.

and Defs contains the following ancestor definition:

new2 $(I, J, N, A, T m p, K):-J<N-I-1, I \geq 0, K \geq 0, J \geq N-I-2, X>W, J>K$, $\operatorname{read}(A, J, W), \operatorname{read}(A, K, X), \operatorname{val}(k, K), \operatorname{val}(j, J), \operatorname{new1}(I, J, N, A, T m p, K)$.

Since the ancestor definition is embedded into the candidate definition via $\subseteq$ or $\cap$ (indeed, the two clauses have the same clause identifier set $\{(\{\mathrm{j}\},\{\}),(\{\mathrm{k}\},\{\})\})$, we obtain a generalization of the candidate definition by applying the widening operator between the linear constraints, hence dropping the constraint $\mathrm{J} \geq \mathrm{N}-\mathrm{I}-2$ of the ancestor definition, and we introduce the following generalized definition:

new4 $(\mathrm{I}, \mathrm{J}, \mathrm{N}, \mathrm{A}, \operatorname{Tmp}, \mathrm{K}):-\mathrm{J}<\mathrm{N}-\mathrm{I}-1, \mathrm{I} \geq 0, \mathrm{~K} \geq 0, \mathrm{X}>\mathrm{W}, \mathrm{J}>\mathrm{K}$, $\operatorname{read}(A, J, W), \operatorname{read}(A, K, X), \operatorname{val}(k, K), \operatorname{val}(j, J), \operatorname{new1}(I, J, N, A, T m p, K)$. 
The correctness of the Transform strategy with respect to the least $\mathcal{A}$-model semantics follows from the correctness results for the unfold/fold rules proved in [18].

The termination of the Transform strategy is based on the following facts. (i) Constraint satisfiability and entailment are checked by a terminating solver (note that completeness is not necessary for the termination of Transform). (ii) The CHR program Arr implementing Constraint Replacement terminates. (iii) The set of new clauses that, during the execution of the Transform strategy, can be introduced by Definition \& Folding steps is finite. Indeed, by construction, they are all of the form $\mathrm{H}:-\mathrm{i}, \mathrm{r}, \mathrm{v}, \mathrm{p}(\mathrm{X})$, where: (1) $\mathrm{X}$ is a tuple of variables, (2) $i$ is an integer constraint, (3) $r$ is a conjunction of array constraints of the form $\operatorname{read}(\mathrm{A}, \mathrm{I}, \mathrm{V})$, where $\mathrm{A}$ is a variable in $\mathrm{X}$ and the variables $\mathrm{I}$ and $\mathrm{V}$ occur in $\mathrm{i}$ only, (4) the set of identifiers of the imperative program is finite, and hence the embedding relation between clauses is a thin well-quasi ordering [19] (this property guarantees that generalization is eventually triggered, and that every definition can be generalized a finite number of times only), (5) the cardinality of $\mathrm{r}$ is bounded, because if in Defs there exists a clause $A$ of the form $\mathrm{H}_{0}:-\mathrm{i}_{0}, \mathrm{r}_{0}, \mathrm{v}_{\mathrm{X}}, \mathrm{p}(\mathrm{X})$, then generalization does not introduce a descendant definition clause $D$ of the form newp (X) : - $i_{\mathrm{X}}, r_{0}, r_{1}, \mathrm{v}_{\mathrm{X}}, \mathrm{p}(\mathrm{X})$ such that $A$ is embedded into $D,(6)$ we assume that the generalization operator on linear constraints has the following finiteness property: only finite chains of generalizations of any given constraint can be generated by applying the operator. The already mentioned generalization operators presented in [10, 19, 40] satisfy this finiteness property. Thus, we have the following result.

Theorem 1. (i) The Transform strategy terminates. (ii) Let program T2 be the output of Transform applied to the input program $T 1$. Then, incorrect $\in M(T 1)$ iff incorrect $\in M(T 2)$.

Let us now conclude our bubblesort-inner example. After a few iterations, the outermost while-loop of the Transform strategy terminates and produces the following set $T 2$ of clauses (which we list as they have been automatically generated):

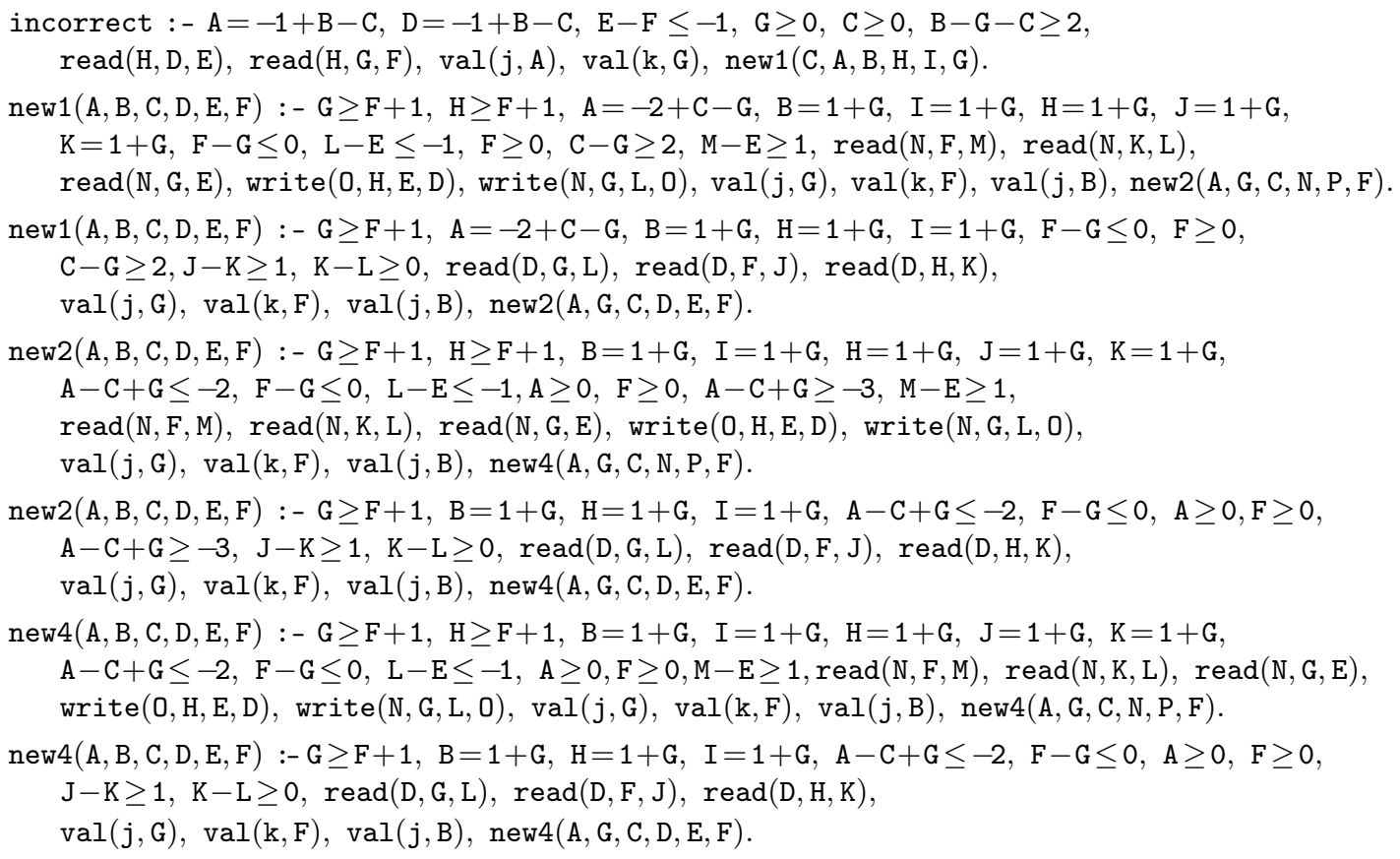

Since this set contains no constrained facts, by Removal of Useless Clauses we remove all clauses from $T 2$ and the Transform strategy outputs the empty program. Thus, incorrect $\notin$ 
$M(T 2)$ and we conclude that the program bubblesort-inner is correct with respect to the given $\varphi_{\text {init }}$ and $\varphi_{\text {error }}$ properties.

\section{Experimental Evaluation}

We have implemented our verification method as a module of the VeriMAP software model checker [15] (available at http://map.uniroma2.it/VeriMAP) and we have performed an experimental evaluation of our method on a benchmark set of programs taken from the literature [6, 9, 16, 27, 35] (the source code is available at http://map.uniroma2.it/smc/ array-chr).

We have applied the Transform strategy presented in Section 3 using different generalization strategies that combine the widening and convex hull operators together with various embedding relations. Different embedding relations are obtained by: (i) selecting different sets of variable identifiers for the introduction of the val constraints, and (ii) using different relations to compare sets of identifiers (see Section 3.3). In particular, we have considered the follow-

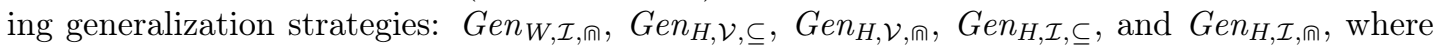
the subscripts should be interpreted as follows. The first subscript denotes the generalization operator: $W$ stands for the widening operator, and $H$ stands for the widening-and-convex-hull operator. The second subscript denotes the selected set of identifiers: $\mathcal{I}$ stands for the set of variable identifiers associated with the second argument (that is, the index) of the read constraints, and $\mathcal{V}$ stands for the set of identifiers associated with the third argument (that is, the value) of the read constraints. The third subscript denotes the relation $r e l \in\{\subseteq$, $\cap\}$ that is used for comparing the sets of identifiers.

The results of our experiments are summarized in Table 1 . The experiments have been performed on an Intel Core Duo E7300 2.66Ghz processor with 4GB of memory under GNU/Linux OS. We have that the strategies based on $G e n_{H, \mathcal{I}, \text { rel }}$ are more precise than those based on $G e n_{H, \mathcal{V}, \text { rel }}$, for any $r e l \in\{\subseteq, \cap\}$. Similarly, the strategies based on $G e n_{H, S, \cap}$ are more precise than those based on $G e n_{H, S, \subseteq}$, for any $S \in\{\mathcal{I}, \mathcal{V}\}$. Note that by generalizing the constraints, the Transform strategy may get an empty set of identifiers associated with a given variable, thereby making the generalizations based on the operator $\subseteq$ less useful that those based on the operator $\mathrm{n}$. The best trade-off between precision and performance is obtained by $G e n_{H, \mathcal{I} \text {, 囘 }}$ that allowed us to prove all programs we have considered. Note also that the bubblesort-inner program can be proved only by generalizations based on $G e n_{W, \mathcal{I}, \text { ก }}$ or $G e n_{H, \mathcal{I}, \text { ก }}$.

\section{$5 \quad$ Related Work and Conclusions}

The technique presented in this paper is an extension of the one presented in [14. The novel contributions of this paper are the following. (1) We have formalized constraint replacement as a CHR program representing the Theory of Arrays, whereas in [14 constraint replacement was implemented directly in CLP. We have shown that the approach based on CHR allows a very elegant combination of constraint manipulation with transformations based on unfold/fold rules. (2) We have presented a novel strategy that controls the generalization of array constraints during CLP transformation by taking into account the information relating the variable identifiers in the imperative program and the CLP representation of their values. We have shown that our generalization strategy is effective on several examples taken from the literature.

In the Introduction we mentioned some CLP-based program verification methods. Here we briefly recall other methods, not based on CLP, for the verification of array programs. 


\begin{tabular}{l||rrrrr}
\hline Program & Gen $_{W, \mathcal{I}, \text { ก }}$ & Gen $_{H, \mathcal{V}, \subseteq}$ & Gen $_{H, \mathcal{V}, \text { ก }}$ & Gen $_{H, \mathcal{I}, \subseteq}$ & Gen $_{H, \mathcal{I}, \text { ก }}$ \\
\hline bubblesort-inner & 0.9 & unknown & unknown & unknown & 1.52 \\
copy-partial & unknown & unknown & 3.52 & 3.51 & 3.54 \\
copy-reverse & unknown & unknown & 5.25 & unknown & 5.23 \\
copy & unknown & unknown & 5.00 & 4.88 & 4.90 \\
find-first-non-null & 0.14 & 0.66 & 0.64 & 0.28 & 0.27 \\
find & 1.04 & 6.53 & 2.35 & 2.33 & 2.29 \\
first-not-null & 0.11 & 0.22 & 0.22 & 0.22 & 0.22 \\
init-backward & unknown & 1.04 & 1.04 & 1.03 & 1.04 \\
init-non-constant & unknown & 2.51 & 2.51 & 2.47 & 2.47 \\
init-partial & unknown & 0.9 & 0.89 & 0.9 & 0.89 \\
init-sequence & unknown & 4.38 & 4.33 & 4.41 & 4.29 \\
init & unknown & 1.00 & 0.97 & 0.98 & 0.98 \\
insertionsort-inner & 0.58 & 2.41 & 2.4 & 2.38 & 2.37 \\
max & unknown & unknown & 0.8 & 0.81 & 0.82 \\
partition & 0.84 & 1.77 & 1.78 & 1.76 & 1.76 \\
rearrange-in-situ & unknown & unknown & 3.06 & 3.01 & 3.03 \\
selectionsort-inner & unknown & time-out & unknown & 2.84 & 2.83 \\
\hline precision & 6 & 10 & 15 & 15 & 17 \\
total time & 3.61 & 21.42 & 34.76 & 31.81 & 38.45 \\
average time & 0.60 & 2.14 & 2.31 & 2.12 & 2.26 \\
\hline
\end{tabular}

Table 1: Verification results using VeriMAP. Time is in seconds. By 'unknown' we indicate that VeriMAP terminates without being able to prove correctness or incorrectness. By 'time-out' we indicate that VeriMAP is unable to provide an answer within 5 minutes.

Some of these methods use abstract interpretation. In [27], which builds upon [24, invariants are discovered by partitioning the arrays into symbolic slices and associating an abstract variable with each slice. A similar approach is followed in 9] where a scalable framework for the automatic analysis of array programs is introduced. In [21, 34] a predicate abstraction for inferring universally quantified properties of array elements is presented, and in [26] the authors present a similar technique which uses template-based quantified abstract domains. In [46] a backward reachability analysis based on predicate abstraction and abstraction refinement is used for verifying assertions which are universally quantified over array indexes.

The methods based on abstract interpretation construct over-approximations, that is, invariants implied by the program executions. These methods have the advantage of being quite efficient because they fix in advance a finite set of basic assertions from which the invariants can be constructed. However, for the same reason, these methods may lack flexibility as the abstraction should be re-designed whenever verification fails.

Also theorem provers have been applied for discovering invariants and proving verification conditions generated from the programs. In particular, in [7] a satisfiability decision procedure for a decidable fragment of a theory of arrays is presented. That fragment is expressive enough to prove properties such as sortedness of arrays. In [32, 33, 38] the authors present some techniques that use theorem proving for generating array invariants. Some theorem proving techniques for program verification are based on Satisfiability Modulo Theory (SMT) (see, for instance, [3, 4, 35]). The approaches based on theorem proving and SMT are more flexible with respect to those based on abstract interpretation because no finite set of assertions is fixed in advance and, instead, the suitable assertions needed for the proofs can be generated on demand.

Although the approach based on CLP program transformation shares many ideas and tech- 
niques with abstract interpretation and automated theorem proving, we believe that it offers a higher degree of flexibility and parametricity. Indeed, the transformation-based method for the generation of the verification conditions and their proof, is to a large extent independent of the imperative program and the property to be verified.

The use of CHR further enhances the flexibility of our transformation-based approach because CHR manipulate the constraints that represent operations on the data structures (such as the read and write operations in the case of arrays), while the unfold/fold rules manipulate the non-constraint atoms of the CLP programs. The experimental results we have reported in this paper demonstrate that the combination of the two kind of rules, those for constraints and those for non-constraint atoms, is a promising, powerful technique for proving program properties.

As future work we plan to extend our transformation-based method to the verification of programs which manipulate dynamic data structures such as lists or heaps. To this aim we may combine the CHR axiomatization of heaps proposed by [17] with the generalization strategies based on widening and convex-hull considered in this paper.

\section{Acknowledgements}

We would like to thank the anonymous referees of VPT 2014 for their helpful comments and constructive criticism.

\section{References}

[1] S. Abdennadher and H. Schütz. CHR ${ }^{\vee}$ : A flexible query language. In Proc. FQAS '98, volume 1495 of $L N C S$, pages 1-14. Springer, 1998.

[2] E. Albert, M. Gómez-Zamalloa, L. Hubert, and G. Puebla. Verification of Java bytecode using analysis and transformation of logic programs. In Proc. PADL 'O\%, volume 4354 of LNCS, pages 124-139. Springer, 2007.

[3] F. Alberti, S. Ghilardi, and N. Sharygina. SAFARI: SMT-based abstraction for arrays with interpolants. In Proc. CAV '12, volume 7358 of $L N C S$, pages 679-685. Springer, 2012.

[4] F. Alberti, S. Ghilardi, and N. Sharygina. Decision procedures for flat array properties. In Proc. TACAS '14, volume 8413 of $L N C S$, pages 15-30. Springer, 2014.

[5] N. Bjørner, K. McMillan, and A. Rybalchenko. Program verification as satisfiability modulo theories. In SMT-COMP '12, pages 3-11, 2012.

[6] N. Bjørner, K. McMillan, and A. Rybalchenko. On solving universally quantified Horn clauses. In Proc. SAS '13, volume 7935 of $L N C S$, pages 105-125. Springer, 2013.

[7] A. R. Bradley, Z. Manna, and H. B. Sipma. What's decidable about arrays? In Proceedings of the 7th International Conference on Verification, Model Checking, and Abstract Interpretation. VMCAI'06, volume 3855 of LNCS, Charleston, SC, USA, January 8-10 2006. Springer.

[8] P. Cousot and R. Cousot. Abstract interpretation: A unified lattice model for static analysis of programs by construction of approximation of fixpoints. In Proceedings of the 4 th ACM-SIGPLAN Symposium on Principles of Programming Languages (POPL'ry), pages 238-252. ACM Press, 1977.

[9] P. Cousot, R. Cousot, and F. Logozzo. A parametric segmentation functor for fully automatic and scalable array content analysis. In Proceedings of the 38th ACM Symposium on Principles of programming languages. POPL'11, pages 105-118, 2011. 
[10] P. Cousot and N. Halbwachs. Automatic discovery of linear restraints among variables of a program. In Proceedings of the Fifth ACM Symposium on Principles of Programming Languages (POPL'78), pages 84-96. ACM Press, 1978.

[11] E. De Angelis, F. Fioravanti, A. Pettorossi, and M. Proietti. Verification of imperative programs by constraint logic program transformation. In Proc. SAIRP '13, volume 129 of EPTCS, pages 186-210, 2013.

[12] E. De Angelis, F. Fioravanti, A. Pettorossi, and M. Proietti. Verifying Programs via Iterated Specialization. In Proceedings of the ACM SIGPLAN 2013 Workshop on Partial Evaluation and Program Manipulation, PEPM '13, pages 43-52, 2013.

[13] E. De Angelis, F. Fioravanti, A. Pettorossi, and M. Proietti. Program verification via iterated specialization. Science of Computer Programming (to appear), 2014. (to appear).

[14] E. De Angelis, F. Fioravanti, A. Pettorossi, and M. Proietti. Verifying array programs by transforming verification. In Proc. VMCAI'14, volume 8318 of LNCS, pages 182-202. Springer, 2014.

[15] E. De Angelis, F. Fioravanti, A. Pettorossi, and M. Proietti. VeriMAP: A tool for verifying programs through transformations. In Proc. TACAS '14, volume 8413 of LNCS, pages 568-574. Springer, 2014.

[16] I. Dillig, T. Dillig, and A. Aiken. Fluid updates: Beyond strong vs. weak updates. In Proc. ESOP '10, volume 6012 of $L N C S$, pages 246-266. Springer, 2010.

[17] G. J. Duck, J. Jaffar, and N. C. H. Koh. Constraint-based program reasoning with heaps and separation. In Proc. CP '13, volume 8124 of $L N C S$, pages 282-298. Springer, 2013.

[18] S. Etalle and M. Gabbrielli. Transformations of CLP modules. Theoretical Computer Science, 166:101-146, 1996.

[19] F. Fioravanti, A. Pettorossi, M. Proietti, and V. Senni. Generalization strategies for the verification of infinite state systems. Theory and Practice of Logic Programming. Special Issue on the 25th Annual GULP Conference, 13(2):175-199, 2013.

[20] C. Flanagan. Automatic software model checking via constraint logic. Sci. Comput. Program., 50(1-3):253-270, 2004.

[21] C. Flanagan and S. Qadeer. Predicate abstraction for software verification. In Proc. POPL '02, pages 191-202. ACM Press, 2002.

[22] T. Frühwirth. Theory and practice of constraint handling rules. Journal of Logic Programming, Special Issue on Constraint Logic Programming, pages 95-138, October 1998.

[23] S. Ghilardi, E. Nicolini, S. Ranise, and D. Zucchelli. Decision procedures for extensions of the theory of arrays. Ann. Math. Artif. Intell., 50(3-4):231-254, 2007.

[24] D. Gopan, T. W. Reps, and S. Sagiv. A framework for numeric analysis of array operations. In Proc. POPL '05, pages 338-350. ACM Press, 2005.

[25] S. Grebenshchikov, A. Gupta, N. P. Lopes, C. Popeea, and A. Rybalchenko. Hsf(c): A software verifier based on horn clauses. In Proc. of the 18th International Conference on Tools and Algorithms for the Construction and Analysis of Systems, TACAS '12, volume 7214 of LNCS, pages 549-551. Springer, 2012.

[26] B. S. Gulavani, S. Chakraborty, A. V. Nori, and S. K. Rajamani. Automatically refining abstract interpretations. In Proc. TACAS '08, volume 4963 of LNCS, pages 443-458. Springer, 2008.

[27] N. Halbwachs and M. Péron. Discovering properties about arrays in simple programs. In Proc. PLDI '08, pages 339-348. ACM Press, 2008.

[28] K. S. Henriksen and J. P. Gallagher. Abstract interpretation of PIC programs through logic programming. In Proc. SCAM '06, pages 103-179, 2006.

[29] J. Jaffar, M. Maher, K. Marriott, and P. Stuckey. The semantics of constraint logic programming. Journal of Logic Programming, 37:1-46, 1998.

[30] J. Jaffar, J. A. Navas, and A. E. Santosa. TRACER: A symbolic execution tool for verification, 2012. http://paella.d1.comp.nus.edu.sg/tracer/ 
[31] J. Jaffar, A. Santosa, and R. Voicu. An interpolation method for CLP traversal. In Proc. CP '09, volume 5732 of $L N C S$, pages 454-469. Springer, 2009.

[32] R. Jhala and K. L. McMillan. Array abstractions from proofs. In Proc. CAV '07, volume 4590 of LNCS, pages 193-206. Springer, 2007.

[33] L. Kovács and A. Voronkov. Finding loop invariants for programs over arrays using a theorem prover. In Proceedings of the 12th International Conference on Fundamental Approaches to Software Engineering. FASE 2009, volume 5503 of LNCS, pages 470-485, 2009.

[34] S. K. Lahiri and R. E. Bryant. Predicate abstraction with indexed predicates. In ACM Trans. Comput. Log., volume 9(1). ACM Press, 2007.

[35] D. Larraz, E. Rodríguez-Carbonell, and A. Rubio. SMT-based array invariant generation. In 14th International Conference on Verification, Model Checking, and Abstract Interpretation, VMCAI 2013, Rome, Italy, January 20-22, 2013, volume 7737 of LNCS, pages 169-188, 2013.

[36] J. W. Lloyd. Foundations of logic programming. Springer-Verlag, Berlin, 1987. Second edition.

[37] J. McCarthy. Towards a mathematical science of computation. In C. Popplewell, editor, Information Processing. Proceedings of IFIP 1962, pages 21-28, Amsterdam, North-Holland, 1963.

[38] K. L. McMillan. Quantified invariant generation using an interpolating saturation prover. In Proc. TACAS '08, volume 4963 of $L N C S$, pages 413-427. Springer, 2008.

[39] M. Méndez-Lojo, J. A. Navas, and M. V. Hermenegildo. A flexible, (C)LP-based approach to the analysis of object-oriented programs. In Proc. LOPSTR '07, volume 4915 of LNCS, pages 154-168. Springer, 2006.

[40] J. C. Peralta and J. P. Gallagher. Convex hull abstractions in specialization of CLP programs. In M. Leuschel, editor, Logic Based Program Synthesis and Tranformation, 12th International Workshop, LOPSTR 2002, Madrid, Spain, September 17-20, 2002, Revised Selected Papers, volume 2664 of $L N C S$, pages $90-108,2003$.

[41] J. C. Peralta, J. P. Gallagher, and H. Saglam. Analysis of Imperative Programs through Analysis of Constraint Logic Programs. In G. Levi, editor, Static Analysis, 5th International Symposium, SAS '98, Pisa, Italy, September 14-16, 1998, volume 1503 of LNCS, pages 246-261. Springer, 1998.

[42] A. Pettorossi and M. Proietti. Transformation of logic programs: Foundations and techniques. Journal of Logic Programming, 19,20:261-320, 1994.

[43] A. Podelski and A. Rybalchenko. ARMC: The Logical Choice for Software Model Checking with Abstraction Refinement. In M. Hanus, editor, Practical Aspects of Declarative Languages, PADL '07, volume 4354 of $L N C S$, pages 245-259. Springer, 2007.

[44] C. J. Reynolds. Theories of Programming Languages. Cambridge University Press, 1998.

[45] P. Rümmer, H. Hojjat, and V. Kuncak. Disjunctive interpolants for Horn-clause verification. In Proc. CAV'13, volume 8044 of LNCS, pages 347-363. Springer, 2013.

[46] M. N. Seghir, A. Podelski, and T. Wies. Abstraction refinement for quantified array assertions. In Proc. SAS '09, volume 5673 of $L N C S$, pages 3-18. Springer, 2009. 